\title{
Effets d'un herbicide et de l'inoculation sur les facteurs de rendement du soja cultivé sur un sol gravillonnaire de plateau
}

\author{
Tamia Joséphine AMA-ABINA ${ }^{1 *}$, Guy Ferrand BEUGRE ${ }^{1}$, Mako François De Paul \\ N'GBESSO ${ }^{2}$, N'Guessan Dominique BROU ${ }^{1}$ et Gballou Réné YORO ${ }^{2}$ \\ ${ }^{1}$ Université d'Abobo-Adjamé; UFR Sciences et Gestion de l'Environnement; Laboratoire de Géosciences et \\ Environnement; 02 BP 801 Abidjan 02, Côte d'Ivoire. \\ ${ }^{2}$ Centre National de Recherche Agronomique; Laboratoire Central Sols Eaux et Plantes; \\ 01 BP 1740 Abidjan 01, Côte d'Ivoire. \\ *Auteur correspondant; E-mail : tamjoseab@yahoo.fr; Tel:(+225) 079716 17/04 573967
}

\section{RESUME}

Dans le cadre de la lutte contre l'enherbement excessif des terres et le maintien de la fertilité de cellesci, une expérimentation sur le soja a eu lieu en zone forestière de Côte d'Ivoire, à la station de recherche du C.N.R.A à Gagnoa pour évaluer l'incidence d'un herbicide et de l'inoculation sur les facteurs de rendement du soja. L'essai s'est tenu sur un sol gravillonnaire de plateau. Une variété (Doko) de soja (Glycine max) et un inoculum, la souche IRAT-FA3 de Bradyrhizobium japonicum ont été utilisés avec le Glyphosate, un herbicide total et systémique appliqué en pré levée, sur un dispositif expérimental en bloc de Fischer à quatre répétitions. Quatre traitements ont été comparés entre eux (T0 : le témoin, T1 : soja traité à l'herbicide, T2: soja inoculé, T3 : soja inoculé traité à l'herbicide). Après trois mois d'essai, les résultats obtenus montrent que l'inoculation a provoqué la nodulation et a amélioré les rendements en biomasse et en graines. Pour les traitements inoculés, on note aussi une forte corrélation positive entre la nodulation et les composantes du rendement. L'herbicide n'a pas eu d'effet néfaste sur la nodulation mais a contribué à améliorer les rendements.

(C) 2012 International Formulae Group. All rights reserved.

Mots clés: rendement, herbicide, inoculation, soja, sol, gravillonnaire.

\section{INTRODUCTION}

L'essor démographique ayant entrâné des besoins alimentaires de plus en plus croissants, la pression sur les terres arables est devenue très forte. Le système traditionnel de gestion des terres qui consiste en une alternance de cultures sur une courte période (3 à 5 ans) et une mise en jachère, sur une période plus longue d'au moins dix ans n'est plus adapté. Aussi, la diminution de la durée de la jachère engendre une baisse de la fertilité et un enherbement excessif des sols
(N'Goran et al., 1997). Face à cette crise des systèmes de culture, des solutions diverses ont été envisagées, parmi lesquelles : l'utilisation des herbicides dans la lutte contre les plantes adventices et l'insertion des légumineuses dans les rotations culturales (Ajaure, 2002 ; Agnoro, 2008 ; Lègère, 2009).

Le soja, l'une de ces légumineuses, a été introduit dans les années 1970 et vulgarisé en Côte d'Ivoire depuis plus de 20 ans à la faveur du projet soja (N'Gbesso et al., 2010). Les travaux de recherche effectués sur le soja 
par l'Institut des Savanes (IDESSA) ont porté sur l'amélioration variétale, les techniques de production et la protection des cultures par la maîtrise des plantes adventices et autres ravageurs.

Pour la maîtrise des plantes adventices, les herbicides ont longtemps constitué des produits clés. Mais leur utilisation répétée pourrait avoir une incidence négative sur les micro-organismes du sol, en particulier, sur les rhizobiums qui assurent la fixation de l'azote atmosphérique en symbiose avec les légumineuses. Les travaux de Gomez et al. (2009) ont montré, à cet effet, que les rhizobiums sont très sensibles aux produits agrochimiques. De plus, selon Brookes (1995) et Saoudi (2008), la fixation symbiotique de l'azote est sensible à la contamination des sols par les métaux lourds. Aussi, la minimisation des risques agro-environnementaux dus à l'utilisation des herbicides nécessite-t-elle une évaluation continue du comportement de ceux-ci sous différents axes d'études.

Cette étude a pour objectif, l'évaluation de l'influence d'un herbicide total sur la nodulation et la croissance du soja cultivé sur un sol gravillonnaire en zone forestière de Côte d'Ivoire.

\section{MATERIEL ET METHODES \\ Matériel \\ Site expérimental}

La station de recherche du C.N.R.A. de Gagnoa a servi de site à notre étude. Elle est située à $5 \mathrm{~km}$ de la ville de Gagnoa $\left(5^{\circ}\right.$ $51^{\prime} \mathrm{W}-6^{\circ} 08 \mathrm{~N}$ ) sur l'axe Gagnoa - Oumé (Figure 1). Le microclimat est caractérisé par deux saisons sèches alternant avec deux saisons pluvieuses. Le premier pic des hauteurs de pluies s'observe en Mai et le second en Octobre. La pluviométrie moyenne annuelle des dix dernières années est de $1520,8 \mathrm{~mm}$ et la température moyenne est de $26{ }^{\circ} \mathrm{C}$ (Figure 2).

Les espèces dominantes rencontrées sur les parcelles sont composées de Mimosacées, de Graminées et de Chromoleana odorata. La parcelle expérimentale est située en haut de versant, sur une pente de $2 \%$. La surface du sol est gravillonnaire avec une forte présence de nodules ferrugineux. L'horizon humifère a une épaisseur de $20 \mathrm{~cm}$. Il est brun jaunâtre foncé (10YR 4/4), sablo argileux avec plus de $50 \%$ de nodules ferrugineux, fragmentaire et poreuse avec de nombreuses racines millimétriques subhorizontales. Il passe progressivement à un horizon, brun vif (7,5YR 5/6) de $13 \mathrm{~cm}$ d'épaisseur, sablo argileux, graveleux (avec plus de 50\% de nodules ferrugineux et de quartz ferruginisé), fragmentaire, poreux avec peu de racines millimétriques subhorizontales. A partir de 33 $\mathrm{cm}$ de profondeur on observe un horizon brun vif $(7,5 \mathrm{YR}$ 5/8), argilo-sableux, moins graveleux (15 à 30\% de nodules ferrugineux et de graviers de quartz), fragmentaire, poreux avec peu de racines millimétriques, subhorizontales (Figure 3).

\section{Matériel végétal et inoculum}

Le matériel végétal utilisé pour cette étude est la variété de soja Doko. Cette variété a un cycle de 95 jours. Elle est à nodulation stricte. Les fleurs sont de couleur blanche et la nodulation est située au collet. La taille des plantes peut atteindre $120 \mathrm{~cm}$ à maturité et le rendement, 2 à 3 t/ha (N'Gbesso et al., 2010).

Pour la production de l'inoculum, Bradyrhizobium japonicum de large répartition géographique et résistante aux métaux lourds (El-Hilali, 2006 ; Chinnaswamy et al., 2008 ; Yan et al., 2011) a été choisie. La souche I.R.A.T-FA3 a été décrite comme étant très compétitive en sol acide et a été déjà utilisée au Rwanda, au Burundi, au Madagascar et au Cameroun (Rolim \& Adou, IDESSA, Bouaké, Côte d'Ivoire, résultats non publiés).

\section{Matériel chimique}

Le Glyphosate communément appelé Round up, un herbicide total et systémique a été utilisé en pré - levée. La dose appliquée aux parcelles élémentaires a été de 4 1/ha.

\section{Méthodes \\ Dispositif expérimental}

Le dispositif expérimental utilisé est un bloc de Fischer avec 4 répétitions. Quatre traitements ont été étudiés. Ce sont :

- Un traitement témoin (T0) dénommé « soja non inoculé sans traitement herbicide »; constitué de semences de soja semées 
directement sans inoculum. Les parcelles n'ont pas été traitées à l'herbicide ;

- Un traitement (T1) «soja non inoculé avec traitement herbicide »; constitué de semences de soja semées sans inoculum. Les parcelles ont été traitées avec l'herbicide au lendemain du semis ;

- Un traitement (T2) «soja inoculé sans traitement herbicide » où les semences de soja ont été mises en contact avec l'inoculum avant d'être semées. Les parcelles n'ont pas été traitées à l'herbicide ;

- Un traitement (T3) «soja inoculé avec traitement herbicide » où les semences de soja ont été inoculées avant le semis. Les parcelles ont été traitées à l'herbicide au lendemain du semis.

Pour chaque répétition, les parcelles élémentaires ont une superficie de $24 \mathrm{~m}^{2}$ dont $15 \mathrm{~m}^{2}$ de parcelle utile.

\section{Inoculation et semis}

Les graines ont été inoculées par enrobage. La dose a été de $100 \mathrm{~g}$ d'inoculum pour $15 \mathrm{~kg}$ de semences soit une densité de $10^{9}$ bactéries par gramme d'inoculum (N'Gbesso et al., 2010). Le sol de la parcelle s. L'herbicide a été appliqué au lendemain du expérimentale est resté au stade de zéro labour et le semis a été immédiatement effectué après l'inoculation, dans des poquets (3 graines de soja par poquets) selon un écartement de 0,5 $\mathrm{m}$ entre les lignes et $0,2 \mathrm{~m}$ sur les lignesemis à l'aide d'un pulvérisateur à dos à la dose de 41 / ha.

\section{Collecte des données végétales}

La nodulation a été mesurée tous les quinze jours, à partir du $30^{\text {ième }}$ jour après semis (JAS) sur les racines de cinq plants prélevés au hasard en bordure des parcelles élémentaires. Les observations ont porté sur l'abondance des nodules en référence à l'échelle d'abondance utilisée par N'Zoué et al. (2003). Cette échelle va de 1 à 5 (1 = pas de nodules; 2 = quelques nodules; $3=1 \mathrm{a}$ moitié des racines porte des nodules ; $4=$ plus de la moitié des racines porte des nodules ; 5 $=$ toutes les racines portent des nodules).

Les quantités de biomasse sèche produites ont été mesurées tous les quinze jours à partir de 60 (JAS). Cinq plants sont prélevés au hasard en bordure des parcelles élémentaires tous les quinze jours, séchés puis, pesés à l'aide d'une balance électronique. Le taux de remplissage des gousses a été déterminé à partir des gousses récoltées sur 20 plants prélevés dans chaque parcelle utile. Les rendements en graines de soja ont été déterminés en pesant les graines récoltées dans chaque parcelle utile.

Les résultats obtenus ont subi une analyse statistique à l'aide du logiciel GENSTAT. Le test utilisé est le test de la LSD au seuil de $5 \%$.

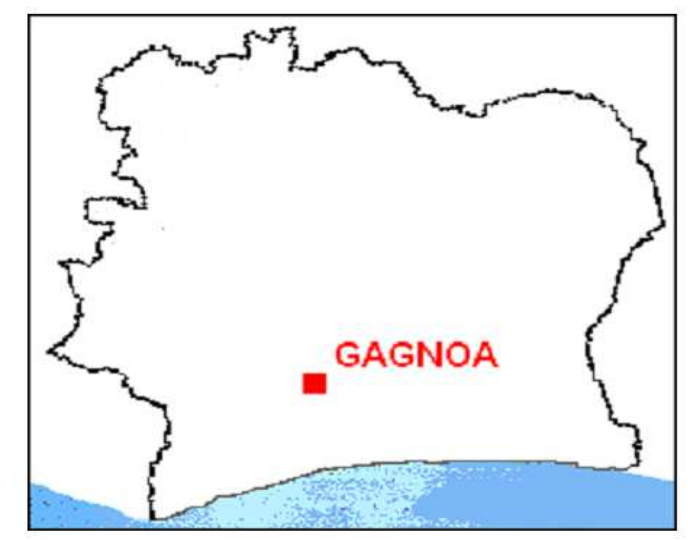

Figure 1: Localisation de la Ville de Gagnoa en Côte d'Ivoire. (Source C.N.R.A., adapté). 


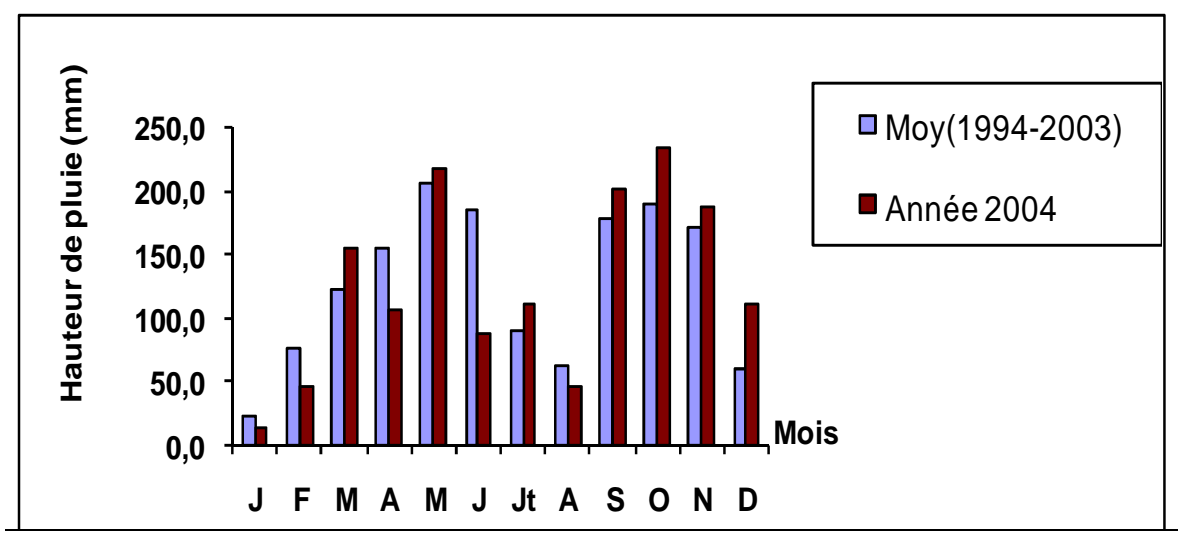

Figure 2: Pluviométrie mensuelle moyenne de la station C.N.R.A. de Gagnoa de 1994 à 2004. (Source : C.N.R.A.).

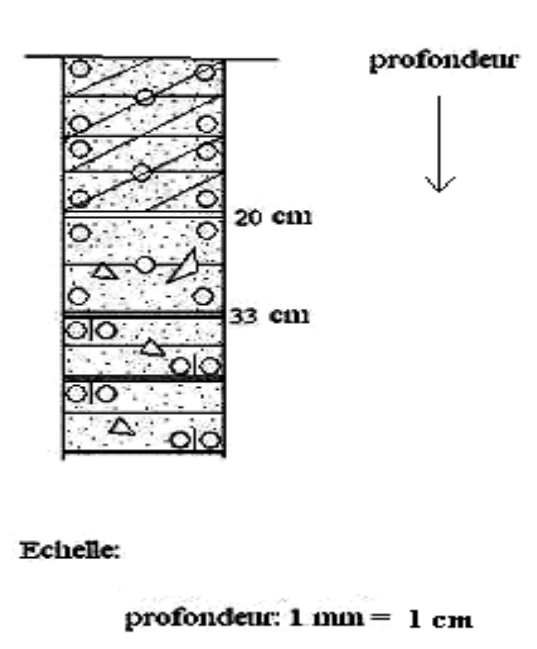

\section{Légende}
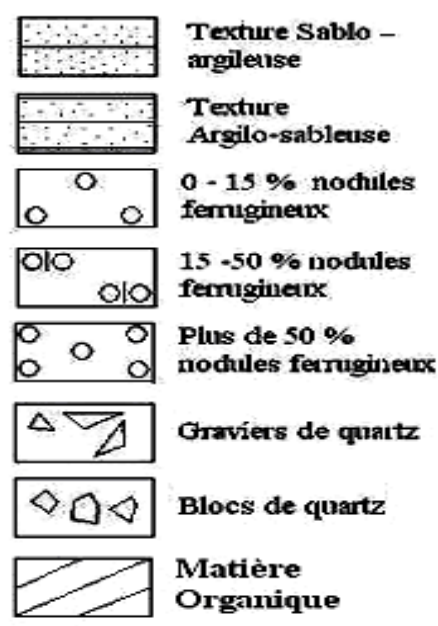

Figure 3: Schéma du profil pédologique du site d'étude.

\section{RESULTATS \\ Nodulation}

La présence des nodules n'a été observée que chez les traitements T2 et T3 avec une abondance moyenne respective de $3,8 / 5$ et $3,65 / 5$. Les valeurs de 1 observées dans le Tableau 1 pour le témoin T0 et le soja non inoculé avec le traitement herbicide T1 signifient qu'aucun nodule n'a été observé au niveau des racines des plants de soja sur les parcelles où ces traitements ont été appliqués. La différence entre les traitements inoculés c'est-à-dire T2, T3 et les traitements non inoculés $\mathrm{T} 0$ et $\mathrm{T} 1$ est hautement significative.
Par ailleurs, à 105 jours après semis (JAS) les abondances de nodules des traitements $\mathrm{T} 2$ $(4,0 / 5)$ et $\mathrm{T} 3(3,1 / 5)$ sont significativement différentes (LSD < 0.05) (Tableau 1).

\section{Quantités de matières sèches}

Les résultats obtenus au niveau de la quantité de matière sèche produite ont montré des différences significatives (LSD $<0.05$ ) entre tous les traitements à 75 et 90 JAS. La plus grande quantité de matière sèche a été obtenue avec les parcelles de soja inoculé avec traitement herbicide $\mathrm{T} 3$ avec une moyenne de 13,44 t/ha. La plus faible quantité 
a été observée chez le témoin avec 5,01 t/ha à 105 (JAS). Ces résultats ont montré que les traitements inoculés T2 et T3 ont produit beaucoup plus de biomasse sèche que les traitements inoculés (Tableau 2).

Taux de remplissage des gousses et rendement en graines

Pour le taux de remplissage des gousses les résultats ne montrent aucune différence significative entre les différents traitements (LSD < 0.05). Toutefois, le plus fort taux de remplissage des gousses a été obtenu dans les parcelles de soja inoculé sans traitement herbicide (T2) avec une moyenne de $68,2 \%$ contre $55,2 \%$ pour le traitement témoin T0. Les traitements $\mathrm{T} 1$ et $\mathrm{T} 3$ ont obtenu respectivement $63,1 \%$ et $64,6 \%$ (Tableau 3).

Au niveau du rendement en graines de soja, l'analyse statistique a montré des différences significatives entre les parcelles des différents traitements. En effet, le rendement le plus élevé $(1,025$ t/ha) a été obtenu dans les parcelles de semences de soja inoculées ayant été traitées à l'herbicide, c'està-dire au niveau du traitement T3. Ensuite, viennent les parcelles des semences de soja inoculées sans traitement herbicide (T2) avec 0,633 t/ha. Enfin, viennent les parcelles des traitements $\mathrm{T} 1$ et $\mathrm{T} 0$ avec seulement 0,426 $\mathrm{t} / \mathrm{ha}$ et $0,326 \mathrm{t} / \mathrm{ha}$, respectivement (Tableau 3).

Par ailleurs, les résultats de l'analyse statistique des données montrent une forte corrélation positive entre la nodulation et les facteurs de rendement à savoir le taux de remplissage des gousses et le poids en grains de soja avec des coefficients de corrélation respectifs de 0.772 et 0.852 (Tableau 4).

Tableau 1: Abondance des nodules en fonction des traitements et du temps de croissance.

\begin{tabular}{lcccccc}
\hline \multirow{2}{*}{ Traitements } & \multicolumn{7}{c}{ Nombre de jours après semis (JAS) } \\
\cline { 2 - 7 } & $\mathbf{3 0}$ & $\mathbf{4 5}$ & $\mathbf{6 0}$ & $\mathbf{7 5}$ & $\mathbf{9 0}$ & $\mathbf{1 0 5}$ \\
\hline T0 & $1,0 \mathrm{~b}^{*}$ & $1,0 \mathrm{~b}$ & $1,0 \mathrm{~b}$ & $1,0 \mathrm{~b}$ & $1,0 \mathrm{~b}$ & $1,0 \mathrm{c}$ \\
T1 & $1,0 \mathrm{~b}$ & $1,0 \mathrm{~b}$ & $1,0 \mathrm{~b}$ & $1,0 \mathrm{~b}$ & $1,0 \mathrm{~b}$ & $1,0 \mathrm{c}$ \\
T2 & $3,1 \mathrm{a}$ & $3,4 \mathrm{a}$ & $3,8 \mathrm{a}$ & $4,1 \mathrm{a}$ & $4,4 \mathrm{a}$ & $4,0 \mathrm{a}$ \\
T3 & $3,0 \mathrm{a}$ & $3,6 \mathrm{a}$ & $3,5 \mathrm{a}$ & $4,3 \mathrm{a}$ & $4,4 \mathrm{a}$ & $3,1 \mathrm{~b}$ \\
\hline LSD (0.05) & 0,47 & 0,71 & 0,40 & 0,57 & 0,61 & 0,63 \\
CV (\%) & 14,4 & 19,6 & 10,8 & 13,7 & 14,2 & 17,2 \\
\hline
\end{tabular}

*Dans chaque colonne, les moyennes suivies de la même lettre ne sont pas significativement différentes.

Seuil de 5\% selon le test de LSD ; LSD : Plus petite différence significative ; CV (\%) : Coefficient de variation

Tableau 2: Quantité de matière sèche (t/ha) produite en fonction du temps par traitement.

\begin{tabular}{lcccc}
\hline \multirow{2}{*}{ Traitements } & \multicolumn{4}{c}{ Nombre de jours après semis $(\mathbf{J A S})$} \\
\cline { 2 - 5 } & $\mathbf{6 0}$ & $\mathbf{7 5}$ & $\mathbf{9 0}$ & $\mathbf{1 0 5}$ \\
\hline T0 & $2,86 \mathrm{a}^{*}$ & $5,10 \mathrm{~b}$ & $7,70 \mathrm{a}$ & $5,01 \mathrm{~b}$ \\
T1 & $2,58 \mathrm{a}$ & $5,40 \mathrm{a}$ & $8,54 \mathrm{a}$ & $8,06 \mathrm{ab}$ \\
T2 & $4,86 \mathrm{a}$ & $8,25 \mathrm{ab}$ & $11,58 \mathrm{a}$ & $8,08 \mathrm{ab}$ \\
T3 & $4,92 \mathrm{a}$ & $10,39 \mathrm{a}$ & $11,96 \mathrm{a}$ & $13,44 \mathrm{a}$ \\
\hline LSD $(0.05)$ & 2,476 & 4,706 & 5,936 & 6,80 \\
CV $(\%)$ & 40,7 & 40,4 & 37,3 & 49,1 \\
\hline
\end{tabular}

*Dans chaque colonne, les moyennes suivies de la même lettre ne sont pas significativement différentes au seuil de 5\% selon le test de LSD ; LSD : Plus petite différence significative ; CV (\%) : Coefficient de variation 
Tableau 3: Taux de remplissage des gousses (G \%) et rendements moyens par traitement en $\mathrm{t} / \mathrm{ha}$.

\begin{tabular}{lcc}
\hline Traitements & G (\%) & Rdt (t/ha) \\
\hline T0 & $55,2 \mathrm{a}^{*}$ & $0,326 \mathrm{~b}$ \\
T1 & $63,1 \mathrm{a}$ & $0,426 \mathrm{~b}$ \\
$\mathrm{~T} 2$ & $68,2 \mathrm{a}$ & $0,633 \mathrm{ab}$ \\
$\mathrm{T} 3$ & $64,6 \mathrm{a}$ & $1,025 \mathrm{a}$ \\
\hline LSD $(0.05)$ & 10,38 & 0,402 \\
CV $(\%)$ & 10,3 & 41,7 \\
\hline *Dans chaque colonne, les moyennes suivies de la même lettre ne sont pas significativement \\
différentes au seuil de 5\% selon le test de LSD; G (\%) : Taux de remplissage des gousses ; \\
Rdt : Rendement en grains du soja ; LSD : Plus petite différence significative; CV $(\%):$ \\
Coefficient de variation
\end{tabular}

Tableau 4: Matrice de corrélation entre la nodulation et les facteurs de rendement du soja.

\begin{tabular}{clll}
\hline & NOD & G (\%) & Rdt \\
\hline NOD & 1 & & \\
G (\%) & 0.772 & 1 & 1 \\
Rdt & 0.825 & 0.584 & 1 \\
\hline
\end{tabular}

NOD : Abondance de nodules; G (\%) : Taux de remplissage des gousses ; Rdt :

Rendement en graines du soja

\section{DISCUSSION}

La présence de nodosités uniquement dans les parcelles inoculées montre que la variété de soja Doko ne nodule pas spontanément. Cette variété est reconnue comme étant une variété à nodulation stricte (N'Gbesso et al., 2010). Les souches de rhizobiums présentes dans le sol du site d'étude ne sont pas infectieuses vis-à-vis de la variété utilisée. Cette propriété, de Bradyrhizobium japonicum à infecter de façon spécifique le soja (Glycine max), a été mentionnée par Sana (2011). En outre, la forte densité des gravillons pourrait avoir influencé l'abondance des nodules en réduisant l'espace disponible entre les concrétions ferrugineuses.

Conformément aux résultats de nombreux auteurs dont Agnoro (2008); Lègère (2009); N'Gbesso et al. (2010), l'inoculation des graines du soja a permis d'obtenir un rendement meilleur grâce à la fixation symbiotique de l'azote. Dans les parcelles inoculées, les plants de soja disposent suffisamment d'azote mis à leur disposition par les bactéries (inoculum) en plus de l'azote disponible dans le sol pour satisfaire leurs besoins nutritifs. Ceci explique la quantité élevée de biomasse sèche produite, le taux de remplissage des gousses ainsi que les rendements élevés chez les traitements T2 et T3 par rapport respectivement aux traitements témoin (T0) et T1. Il en serait de même de la forte corrélation positive entre la nodulation et le taux de remplissage des gousses ainsi que du rendement en grains. Cependant, les rendements de 326 à 1025 $\mathrm{kg} / \mathrm{ha}$ obtenus sont nettement inférieurs à ceux mentionnés pour la même variété Doko (2 à 3 t/ha) dans les travaux d'évaluation variétale effectués par N'Gbesso et Konaté (2000) à 
Touba, Odienné et Bouaké. Cela pourrait être dû au fait que Gagnoa n'est pas la zone écologique favorable du soja. Ces différences pourraient aussi s'expliquer par la nature graveleuse du sol et par la mauvaise répartition de la pluviométrie pendant la période de l'essai, entraînant un manque d'eau aux stades critiques du développement des plants (floraison, formation et remplissage de gousses).

L'horizon humifère du sol de la parcelle expérimentale a un taux pondéral en éléments grossiers très élevé (plus de 50\% par endroit). Ce taux élevé en éléments grossiers réduit le volume de la fraction argileuse du sol et la quantité des éléments nutritifs adsorbables par les racines des plantes (Boa, 1990 ; Yoro et al., 1995), rendant ainsi faible la réserve en eau utile. Ces résultats sont en conformité avec ceux de Berk (1993) qui souligne que pour la culture du soja, les sols à faible capacité de rétention en eau produisent peu.

L'effet bénéfique de l'application de l'herbicide, observé à travers les paramètres étudiés, pourrait s'expliquer par le fait que son action sur les plantes adventices a permis de réduire la compétition par rapport à l'espace, à la lumière, à l'eau et aux éléments nutritifs du sol. Cette réduction de la compétition serait à la base des bons rendements observés chez les traitements $\mathrm{T} 1$ et $\mathrm{T} 3$, par rapport au témoin. La pression des plantes adventices a été donc plus forte dans les parcelles non traitées à l'herbicide; ce qui pourrait être à la base des faibles valeurs de rendements du traitement témoin (T0) par rapport au traitement T1. Ces résultats sont en conformité avec les observations faites par plusieurs auteurs dont Schmid et al. (1983); Peeters et Salembier (1995) qui ont mis en relief les effets négatifs de la pression des mauvaises herbes sur les cultures et leurs rendements et ont préconisés l'utilisation des herbicides pour améliorer les récoltes.

L'application de l'herbicide et l'inoculation ont ensemble, permis d'obtenir les meilleurs rendements. L'herbicide n'a pas eu d'effet significatif sur le nombre de nodules formés. Cela pourrait être la conséquence de la période d'application de l'herbicide (pré levée), qui s'est en grande partie décomposé avant la formation des premiers nodules sur les racines des plants de soja. Ces résultats corroborent ceux de Anikwé et al. (2003). La baisse de l'abondance des nodules, observée à 105 JAS pour les traitements T2 et T3, pourrait être due à une perte de nodules lors de l'échantillonnage. Le fait que l'abondance de nodules et le taux de remplissage des gousses, observés chez le traitement $\mathrm{T} 2$, soient supérieurs à ceux observés chez le traitement $\mathrm{T} 3$, alors que le rendement moyen observé chez le traitement $\mathrm{T} 3$ est supérieur à celui du traitement $\mathrm{T} 2$, serait dû à un phénomène d'avortement ou de malformation des graines formées chez le traitement T2. Ceci est la conséquence de l'application de l'herbicide qui a réduit la compétition à la nutrition hydrique et minérale chez le traitement $\mathrm{T} 3$ au bénéfice de l'efficience des nodules. L'herbicide ou les résidus issus de sa décomposition ont probablement stimulé l'activité fixatrice d'azote, notamment celle de la nitrogénase comme l'a signalé Dick (1998) qui a, par ailleurs, observé que l'application d'un herbicide à la dose prescrite, stimule l'activité de la déshydrogénase et de l'uréase dans le sol.

\section{Conclusion}

L'étude menée, a permis d'identifier les effets de l'inoculation sur les rendements du soja. Celle-ci a permis d'obtenir des quantités de biomasse et des rendements en graines élevés par rapport au soja non inoculé. Cette amélioration est due aux retombées de la fixation symbiotique de l'azote atmosphérique. Toutefois les performances escomptées n'ont pu être réalisées à cause du taux de gravillons du sol et des perturbations enregistrées au niveau de la pluviométrie. Ce 
qui a créé un déficit hydrique à certains stades du développement des plantes.

Cette expérimentation a aussi mis en évidence, des effets bénéfiques de l'herbicide. Par son mode d'action sur les plantes adventices, l'herbicide a permis de réduire la compétition hydrique et minérale entre le soja et les mauvaises herbes. Cela a aussi favorisé la production en biomasse et en graines. L'application de l'herbicide n'a pas eu d'effet négatif sur la formation des nodules. En outre, l'efficience des nodules formés s'est vue renforcée.

Les recherches, de plus longues durées, devront se poursuivre pour la détermination de l'influence des facteurs pédoclimatiques sur les rendements du soja et la maîtrise des effets des herbicides en fonction des types de sols en ayant un regard appuyé sur les caractères physiques et morphologiques du sol. Des doses minimales d'herbicide tolérables par les rhizobiums et ayant un faible impact agro-environnemental seront ainsi déterminées pour l'amélioration de la culture du soja en Côte d'Ivoire.

\section{REFERENCES}

Agnoro M. 2008. Effet de l'inoculation avec "bradyrhizobium japonicum" et de l'apport de phosphore sur la productivité du soja (glycine max) en champs paysans au Benin. Oléoscope, 66: 27-28.

Anikwé MAN, Okonkwo CI, Mbah CN. 2003. Nodulation effectivity, N-Accumulation and yield of soybean treated with Pre- and Post-Emergence herbicides. Tropicultura, 21(1): 22 - 28.

Berk Z. 1993. Technologie de production de farine alimentaire et de produits protéiques issus du soja. Bulletin des Services Agricoles FAO 37.

Boa D. 1990. Principales Aptitudes et Contraintes des Sols du Bassin. L'Importance des Gravillons : Structure et Fonctionnement Hydro Pédologique d'un Petit Bassin Versant de Savane Humide
(Etudes et Thèses, ) Edition de l'ORSTOM: Paris; 233-245.

Brookes PC. 1995. The use of microbial parameters in monitoring soil pollution by heavy metals. Biol. Fert. Soils, 19: 269279.

Chinnaswamy A, Angèle NZ, and Gisèle L. 2008. Genetic Diversity of Native Bradyrhizobia Isolated from Soybeans (Glycine max L.) In Different AgriculturalEcological-Climatic Regions of India. Appl. Environ. Microbiol., 74(19): 59915996.

Dick RP. 1998. Soil enzyme activities as integrative indicators of soil health. Biological Indicators of Soil Health. CAB International, 121-157.

El-Hilali I. 2006. La symbiose Rhizobium Lupin: Biodiversité des micro-symbiotes et mise en évidence d'une multi infection nodulaire chez Lupinus luteus. Thèse Doctorat, Université Mohammed V. Agdal, Rabat.

Gomez E, Ferreras L, Lovotti L, Fernandez E. 2009. Impact of glyphosate application on microbial biomass and metabolic activity in a Vertic Argiudoll from Argentina. Eur. J. Soil., 45: 163-167.

Lègère A. 2009. Biodiversité et productivité utopie ou réalité ? Constat après 18 années de pratiques de conservation des sols. Colloque sur la Phyto-protection 22 oct. 2009.

N'Gbesso MF, Konaté D. 2000. Rapport d'activités de la convention CNRA/Projet Soja. C.N.R.A: Bouaké, 62 p.

N'gbesso MF, N'guetta ASP, Kouamé N, Foua BK. 2010. Evaluation de l'efficience de l'inoculation des semences chez 11 génotypes de soja (Glycine max L. Merril) en zone de savane de Côte d'Ivoire. Sci. Nat., 7(1): 1812-0741.

N'Goran A, Gnahoua GM, Oualou K, Pity P. 1997. Evolution de la fertilité d'un sol au cours de 4 ans de culture, suite à une jachère de 6 ans. Cas d'une zone de forêt humide en Côte d'Ivoire: Jachères et 
maintien de la fertilité. Actes de l'Atelier de Bamako, 101-106.

N'Zoué A, Kouamé C, Mondeil F, N'Gbesso M. 2003. Analyse agro morphologique de deux lignées de soja (Glycine max L. Merril). Agronomie Africaine, 15(3): 93104.

Peeters A, Salembier JF. 1995. Contrôle des Mauvaises Herbes; Base Physiologique et Agronomique de la Production Végétale: Agronomie Moderne. Hatier-AUPELFUREF; 427-464.

Sana DF. 2011. Diversité phénotypique et moléculaire des micros symbiotes du Sulla du nord (Hedysarum coronarium L.) et sélection de souches rhizobiales efficientes. Thèse de Doctorat, Sci.-Agro, INA, Tunisie.

Saoudi M. 2008. Les bactéries nodulant les légumineuses (B.N. ; L.P.) : Caractérisation des bactéries associées aux nodules de la légumineuse Astragalus armatus. Mémoire Magister, Université de Constantine.

Schmid W, Dossekou M, Koch WA, Walter H. 1983. Aspect de modification et nuisibilité de la flore adventice dans les systèmes au Togo. C. R. de la $2^{\text {ième }}$ Conférence Bis. SOAM, Abidjan, Côte d'Ivoire, 19-33.

Yan MZ, Ying L, Wen FC, En TW, Chang FT, Qin QL, Yun ZZ, Xin HS and Wen XC. 2011. Biodiversity and Biogeography of Rhizobia Associated with Soybean Plants Grown in the North China Plain. Appl. Environ. Microbiol., 77(18): 63316342.

Yoro G, Ama TJ, Gnohouri G. 1995. Technical report on land development for sustainable agriculture in Côte d'Ivoire. IBSRAM-IDEFOR/DCC; 96. 